\title{
A SURVEY ON FACTORS THAT IMPACT INDUSTRIAL AGENT ACCEPTANCE
}

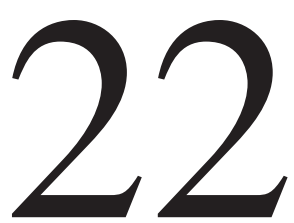

\author{
Paulo Leitão $0^{1,2}$ and Stamatis Karnouskos ${ }^{3}$ \\ ${ }^{1}$ Polytechnic Institute of Bragança, Bragança, Portugal, \\ ${ }^{2}$ LIACC-Artificial Intelligence and Computer Science Laboratory, Porto, Portugal \\ ${ }^{3} \mathrm{SAP}$, Karlsruhe, Germany
}

\subsection{INTRODUCTION}

Agent technology provides a set of interesting characteristics such as modularity, flexibility, robustness, reconfigurability, and responsiveness based on the decentralization of control functions over a plethora of distributed, autonomous, and cooperative agents, constituting an alternative way to design, simulate, and realize complex engineering systems. These concepts were initially applied to business, electronic commerce, and management systems, but their application to industrial domains, such as manufacturing, logistics, telecommunications, smart grids, and health care have also been reported in the literature, and particularly in several survey papers (Pechoucek and Marik, 2008; Leitão, 2009; Leitão et al., 2013).

At this stage, it is important to clarify the difference between traditional software agents and industrial agents (IA). In fact, industrial agents inherit software agent principles, such as intelligence, autonomy, and cooperation, which are applicable to industrial applications, and then face industrial requirements (Göhner, 2013; Pereira et al., 2012), such as hardware integration, reliability, faulttolerance, scalability, industrial standard compliance, quality assurance, resilience, manageability, and maintainability. Depending on the scenario in which industrial agents are used, these requirements may have varying degrees of importance. However, the focus is on well-established, stable, and proven approaches rather than experimental and not fully tested features. Also, industrial solutions need to fully guarantee business continuity, as well as compliance to the legal requirements posed on the industry. Hence, technology as such is not the only criterion. The whole operational context and life cycle of the solution is considered.

At this moment, more than 10 years after the first industrial application of agent technology, namely in a production line for producing cylinder heads for diesel engines at the Daimler-Chrysler factory plant in Stuttgart, Germany (Schild and Bussmann, 2007), it is important to analyze the current level of adoption of agent technology in industrial environments. In the literature, several survey review papers were published analyzing the level of adoption of agent technology in industrial environments (Marik and McFarlane, 2005; McFarlane and Bussmann, 2000; Monostori et al., 2006; Shen et al., 2006; Babiceanu and Chen, 2006; Marik and Lazansky, 2007; Pechoucek and Marik, 2008; Leitão, 2009; Leitão et al., 2013). These surveys mainly focused on the existing developments by describing 
the agent-based solutions deployed in industry and discussing possible roadblocks for a wider adoption by industry stakeholders.

Several efforts were made in these last years to advance the domain of agents and more specifically introduce them into real-world industrial settings. The European Commission funded Agentlink III coordination action for agent-based computing promoted in 2005 a strategic roadmap in agent technology aiming to analyze the past and current state of agent technologies, and identify the challenges and obstacles that need to be tackled for the higher commercial adoption of the technology (Luck et al., 2005). In Germany, the VDI/VDE GMA Technical Committee 5.15 on Multi-agent Systems also surveyed the use of multi-agent systems in industrial automation (VDI/VDE, 2653, 2010; Göhner, 2013). Under the scope of the research project KREAagentuse (2014), a tool-supported method for the development of agent systems in automation technology was evaluated by the project partner Beckhoff Automation $\mathrm{GmbH}$ and its customers, taking into account the usability aspects. A look also at the Gartner (2014) hype cycle for emerging technologies reveals agent-related technologies in various phases, such as analytics, M2M communication, gamification, cloud computing, big data, the Internet of Things, autonomous vehicles, smart advisors, data science, predictive analytics, and smart robots.

However, all these efforts miss a deeper analysis of the factors and criteria that constrain the real and "massive" industrial adoption of agent technology, their impact on the technology's acceptance, and the actions to be performed to overcome the current situation. For this purpose, the IEEE Industrial Electronics Society Technical Committee on Industrial Agents (http://tcia.ieee-ies.org) set up a survey to analyze the factors that impact the industrial adoption of agent technology, considering the valuable expert knowledge in industrial agents' domains by experts coming from industry and academia.

This chapter is organized as follows: Section 22.2 discusses the theoretical aspects behind the selection of the factors to be analyzed in the survey; Section 22.3 presents some insights on the methodology used to perform the survey; and Section 22.4 forms the core of this chapter, introducing survey results and analysis. Finally, Section 22.5 presents the conclusions of the survey, including a summary of the results using a SWOT (Strengths, Weaknesses, Opportunities, and Threats) analysis.

\subsection{FACTORS FOR INDUSTRIAL AGENT ACCEPTANCE}

As pointed out by Marik and McFarlane (2005), in spite of the promising perspective of the multi-agent systems approach, the industrial applications of control systems based on its principles are rare, and the implemented functionalities are normally restricted, with industry being very slow in adopting these concepts.

The pertinent question that arises is why the use of agent technology is not more widely adopted by industry, especially considering research efforts of the last decades. The answer is neither clear, nor made up of one simple answer. Some possible barriers to large-scale dissemination in industrial environments were identified and discussed in several survey review papers, namely in Pechoucek and Marik (2008) and Leitão (2009). As suggested by Leitão (2009), two groups of reasons can be identified:

- Conceptual efficiency in the paradigm, including aspects such as distributed thinking as a new way to design these systems, the demand of industry for proven and mature technology, the use of self-* algorithms to support reconfigurability, interoperability as a key issue in the development of distributed and heterogeneous systems, and the integration of physical automation devices (normally tens or hundreds) with the software control system. 
- Development-related aspects, including issues such as methodologies that formalize the structure and behavior of these systems, and the use of scalable and robust development platforms to address industrial requirements.

Marik and McFarlane (2005) and Leitão et al. (2013) also point out other reasons, such as the investment to set up these solutions and the compliance with standards to fulfill the industrial requirements. Additionally, as stated in the Agent Link roadmap (Luck et al., 2005), the agent technology in 2005 was still in its infancy, and the agent programming skills are not yet widespread among commercial software developers.

The referred identified reasons were analyzed and mapped into a set of selected factors that may have an impact on the acceptance of industrial agents, to be further analyzed during the survey process. As depicted in Figure 22.1, these consist of: design, technology, intelligence/algorithms, hardware, cost, standardization, application, and challenges.

Industrial agents offer a new and alternative way to design complex reconfigurable systems based on the decentralization of functions, following the principle of divide and conquer. The set of questions within the design factor will analyze the requirements for the design of such systems, namely the importance of robustness, fault-tolerance, interoperability, scalability, security, and modularity properties.

The availability of mature tools and development methodologies that simplifies the engineering of agent-based systems (integrating the design, verification, simulation, and deployment phases) are crucial issues to be analyzed and may impact the acceptance of the agent technology. For this purpose, the topic regarding technology factor analyzes, among others, the use of integrated development environment (IDE) tools and proven Internet technologies, and the avoidance of proprietary technologies to enable a wider use of the industrial agents concept.

Software agents may embed intelligent and self-* algorithms, probably derived from the artificial intelligence (AI) field and/or inspired from biology, which properly regulate their behaviors to achieve their individual goals, contributing to the emergence of the system's objectives. The topic regarding the

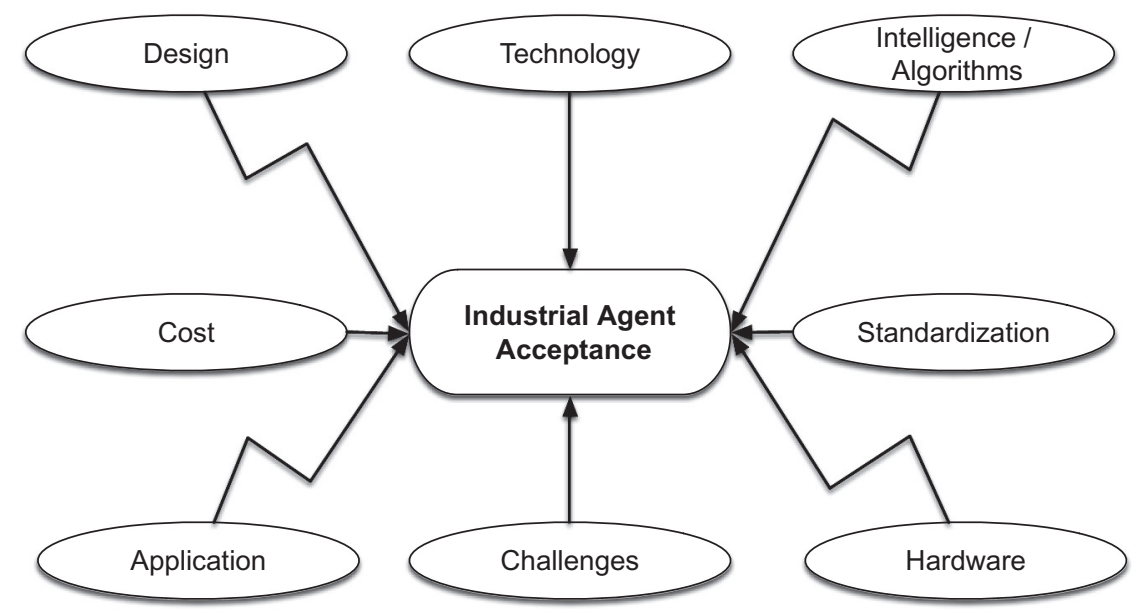

FIGURE 22.1

Overview of industrial agent acceptance factors. 
intelligencelalgorithms factor analyzes how the use of new and effective algorithms may contribute to the success of industrial agent solutions.

Industrial agent-based systems are comprised of software agents managing and/or operating in physical hardware devices. The integration of these two components is usually performed case by case, requiring the implementation of a huge and expensive effort, where methodologies or standard interfaces are sought that support the easy, fast, transparent, and reusable integration of physical automation devices. The group of questions associated with the hardware factor handles the way physical hardware devices can be integrated with agent technology to form a coherent cyber-physical system, as well as deliver dependent applications and services (Colombo et al., 2014; Höller et al., 2014).

The cost associated with the development, operation, and maintenance of the agent-based solutions, and particularly the return on investment (ROI), needs a deep analysis. In fact, the use of agent technology requires an immediate investment, and the return usually only appears much later in the future when the need for adaptation and reconfiguration occurs. For this purpose, the topic related to the cost factor analyzes the different dimensions of the cost to develop an industrial agent-based solution, namely covering the hardware, software development, migration, maintenance, and training perspectives.

The standardization issue is pointed out by industry experts as a major challenge for the industrial acceptance of the agent technology, impacting the adoption of this technology. The Foundation for Intelligent Physical Agents (FIPA) defines a set of standards for the development of multi-agent systems, but it misses many requirements and particularities imposed by industrial environments, which require a deeper analysis. For this purpose, a set of questions within the standardization factor analyzes the need for industrial agent solutions to be compliant with existing automation and IT standards and explores the need to establish new and proper standards in the field.

Industrial agents can be applied to different domains, but the expected benefits may depend on the requirements and constraints imposed by the type of application domain to be considered. The set of questions associated with the application factor analyzes the use of agent technology in applications ranging from simulation to real-time control, including intermediate levels such as the reconfiguration of assets.

The analysis of the challenges is of critical importance to design actions for a wider adoption of the agent technology. The topic regarding the challenges factor analyzes several possible roadblocks that constrain a wider adoption of industrial agents, as well the challenges that should be considered to increase their acceptance.

\subsection{METHODOLOGY, DATA COLLECTION, AND DEMOGRAPHICS}

The implemented survey is based on a structured questionnaire that compiles information from the experience and knowledge of the participants, the majority of which were targeted to be experts in the agent domain, including users, developers, and managers. The construction of this survey is based (i) on the results of previous surveys conducted, and (ii) its guiding focus was the factors that impact industrial agent acceptance. More specifically, we have considered survey outcomes carried out by Müller and Fischer (2014), KREAagentuse (2014), and Leitão (2009). As such, this survey can be considered as complementary to the existing ones and may act as the starting point for more in-depth research on the aspects identified. 
The collection of data was realized online via a survey form that considered clear, concrete, and precise questions, formulating alternative choices that guarantee logical and possible answers. For this purpose, the survey was constructed to collect answers per factor in one page, and then move to the next one while also providing a bar indicator showing how much of the survey was completed. In each step, the user was prompted if missing or invalid data was entered in order to make sure that the collected data per step were full and also semantically valid. In addition, a free text area permitted additional input from users. The questionnaire responses were anonymous. Due to the way the data collection was done, we had guaranteed that there would be no missing data. The resulting dataset comprises 118 valid answers, which are assessed in this chapter.

Table 22.1 depicts the descriptive statistics for the defined variables, which represent the questions in the survey-i.e., D1-D8 (Design), T1-T5 (Technology), H1-H4 (Hardware), I1-I5 (Intelligence/ Algorithms), C1-C7 (Cost), S1-S6 (Standardization), A1-A6 (Application), CH1-CH8 (Challenges), IAA1-IAA6 (Industrial agents acceptance). We had 118 valid answers $(n=118)$ and the mean, standard deviation (sd), min, max, skewness (skew), and kurtosis are depicted. As we can see, all variables

\begin{tabular}{|l|l|l|l|l|l|l|l|}
\hline Table 22.1 Descriptive statistics of the survey dataset \\
vars & n & mean & sd & min & max & skew & kurtosis \\
D1 & 118 & 4.22 & 0.82 & 1 & 5 & -1.19 & 1.89 \\
D2 & 118 & 4.11 & 0.79 & 1 & 5 & -1.04 & 1.83 \\
D3 & 118 & 4.26 & 0.86 & 1 & 5 & -1.35 & 1.94 \\
D4 & 118 & 4.25 & 0.79 & 1 & 5 & -1.13 & 1.90 \\
D5 & 118 & 4.07 & 1.00 & 1 & 5 & -1.33 & 1.85 \\
D6 & 118 & 3.86 & 0.87 & 1 & 5 & -1.10 & 1.90 \\
D7 & 118 & 4.19 & 0.90 & 1 & 5 & -1.33 & 1.99 \\
D8 & 118 & 4.06 & 0.97 & 1 & 5 & -1.31 & 1.85 \\
T1 & 118 & 3.55 & 1.00 & 1 & 5 & -0.97 & 0.87 \\
T2 & 118 & 4.01 & 0.89 & 1 & 5 & -1.05 & 1.36 \\
T3 & 118 & 3.66 & 1.16 & 1 & 5 & -0.70 & -0.32 \\
T4 & 118 & 3.45 & 1.14 & 1 & 5 & -0.67 & -0.16 \\
T5 & 118 & 4.01 & 0.94 & 1 & 5 & -1.15 & 1.50 \\
H1 & 118 & 3.75 & 0.98 & 1 & 5 & -1.33 & 1.86 \\
H2 & 118 & 3.41 & 1.03 & 1 & 5 & -0.65 & 0.12 \\
H3 & 118 & 3.76 & 0.97 & 1 & 5 & -1.06 & 1.47 \\
H4 & 118 & 3.84 & 0.91 & 1 & 5 & -1.15 & 1.86 \\
I1 & 118 & 3.96 & 1.07 & 1 & 5 & -1.14 & 0.81 \\
I2 & 118 & 4.06 & 1.02 & 1 & 5 & -1.29 & 1.36 \\
I3 & 118 & 3.83 & 1.04 & 1 & 5 & -0.95 & 0.61 \\
I4 & 118 & 4.06 & 1.05 & 1 & 5 & -1.07 & 0.53 \\
I5 & 118 & 3.84 & 1.15 & 1 & 5 & -1.10 & 0.53 \\
C1 & 118 & 3.59 & 0.98 & 1 & 5 & -0.93 & 0.98 \\
C2 & 118 & 3.83 & 0.81 & 1 & 5 & -0.96 & 1.77 \\
\hline
\end{tabular}




\begin{tabular}{|c|c|c|c|c|c|c|c|}
\hline vars & $\mathbf{n}$ & mean & sd & $\min$ & $\max$ & skew & kurtosis \\
\hline C3 & 118 & 3.58 & 0.99 & 1 & 5 & -0.94 & 1.05 \\
\hline $\mathrm{C} 4$ & 118 & 3.79 & 1.00 & 1 & 5 & -1.01 & 1.12 \\
\hline C5 & 118 & 3.42 & 1.07 & 1 & 5 & -0.56 & 0.02 \\
\hline C6 & 118 & 3.47 & 1.01 & 1 & 5 & -0.76 & 0.50 \\
\hline $\mathrm{C} 7$ & 118 & 3.39 & 0.95 & 1 & 5 & -1.04 & 0.92 \\
\hline S1 & 118 & 3.87 & 1.05 & 1 & 5 & -1.05 & 0.91 \\
\hline S2 & 118 & 3.69 & 1.10 & 1 & 5 & -0.79 & 0.18 \\
\hline S3 & 118 & 3.74 & 1.03 & 1 & 5 & -0.92 & 0.86 \\
\hline S4 & 118 & 3.74 & 1.05 & 1 & 5 & -0.90 & 0.70 \\
\hline S5 & 118 & 3.85 & 1.05 & 1 & 5 & -0.99 & 0.79 \\
\hline S6 & 118 & 3.49 & 1.07 & 1 & 5 & -0.58 & -0.09 \\
\hline A1 & 118 & 4.03 & 0.91 & 1 & 5 & -1.23 & 1.91 \\
\hline A2 & 118 & 4.14 & 0.82 & 1 & 5 & -1.12 & 1.83 \\
\hline A4 & 118 & 3.78 & 1.02 & 1 & 5 & -0.96 & 0.88 \\
\hline A3 & 118 & 3.80 & 1.06 & 1 & 5 & -0.90 & 0.55 \\
\hline A5 & 118 & 3.92 & 1.11 & 1 & 5 & -0.99 & 0.45 \\
\hline A6 & 118 & 3.56 & 1.02 & 1 & 5 & -0.86 & 0.71 \\
\hline $\mathrm{CH} 1$ & 118 & 3.46 & 1.08 & 1 & 5 & -0.66 & 0.09 \\
\hline $\mathrm{CH} 2$ & 118 & 3.53 & 1.17 & 1 & 5 & -0.63 & -0.39 \\
\hline $\mathrm{CH} 3$ & 118 & 3.55 & 1.14 & 1 & 5 & -0.75 & 0.02 \\
\hline $\mathrm{CH} 4$ & 118 & 3.59 & 1.09 & 1 & 5 & -0.75 & 0.19 \\
\hline CH5 & 118 & 3.59 & 1.16 & 1 & 5 & -0.60 & -0.31 \\
\hline CH6 & 118 & 3.52 & 1.11 & 1 & 5 & -0.64 & -0.14 \\
\hline $\mathrm{CH} 7$ & 118 & 3.47 & 1.14 & 1 & 5 & -0.65 & -0.12 \\
\hline CH8 & 118 & 3.31 & 1.16 & 1 & 5 & -0.37 & -0.62 \\
\hline IAA1 & 118 & 4.11 & 0.97 & 1 & 5 & -1.32 & 1.92 \\
\hline IAA2 & 118 & 3.79 & 1.01 & 1 & 5 & -1.02 & 1.06 \\
\hline IAA3 & 118 & 3.85 & 0.97 & 1 & 5 & -1.19 & 1.85 \\
\hline IAA4 & 118 & 3.76 & 1.05 & 1 & 5 & -0.94 & 0.78 \\
\hline IAA5 & 118 & 3.74 & 0.97 & 1 & 5 & -1.09 & 1.60 \\
\hline IAA6 & 118 & 3.58 & 1.06 & 1 & 5 & -0.71 & 0.20 \\
\hline
\end{tabular}

exhaust the available Likert value space. The mean values reflect the central tendency of our data, while the dispersion around the mean is shown in the standard deviation column.

As can be seen in Figure 22.2, the background of the survey responders covers a large spectrum, coming mostly from the university sector, but also from industry, small/medium enterprises (SMEs), research centers, non-profit organizations, and government. Although the percentage of university participants is very high, this is not seen as problematic because professors and researchers are heavily involved in cutting-edge research on industrial agents and hence the views depicted are relevant. As can be also witnessed from the organizational position, the large majority of the participants are professionals-i.e., managers, engineers/developers, researchers, and professors. 


\section{Professional Domain}

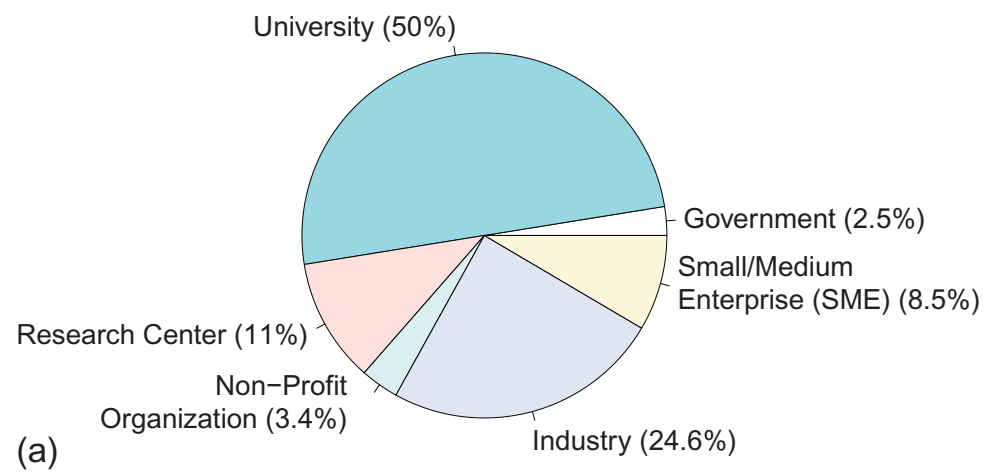

\section{Organizational Position}

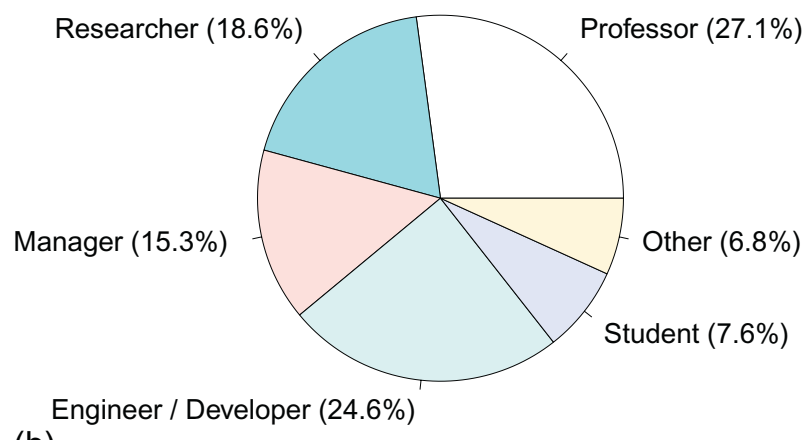

(b)

FIGURE 22.2

Survey demographics: background.

As illustrated in Figure 22.3, the majority of the survey participants have over 10 years of professional expertise, while an additional one-third also have significant professional expertise-i.e., 4-9 years. In addition, it is also evident that the agent-relevant expertise is substantial, with more than $80 \%$ percent of participants having several years of expertise in various aspects of agent concepts and technologies.

These demographics are not surprising because the survey has been disseminated among the relevant stakeholders of IEEE IES Technical Committee on Industrial Agents (http://tcia.ieee-ies.org), as well as relevant conferences, workshops, and agent development mailing lists. As such, the results presented here seem to correspond to a big part of the industrial agents community.

\subsection{SURVEY RESULTS AND ANALYSIS}

The survey uses the Likert scale for all answers-i.e., (1) Strongly Disagree, (2) Disagree, (3) Neutral (neither agree nor disagree), (4) Agree, and (5) Strongly Agree. The figures per factor display the answers and their percentages (note that all numbers are rounded). In addition to sum percentages for the 


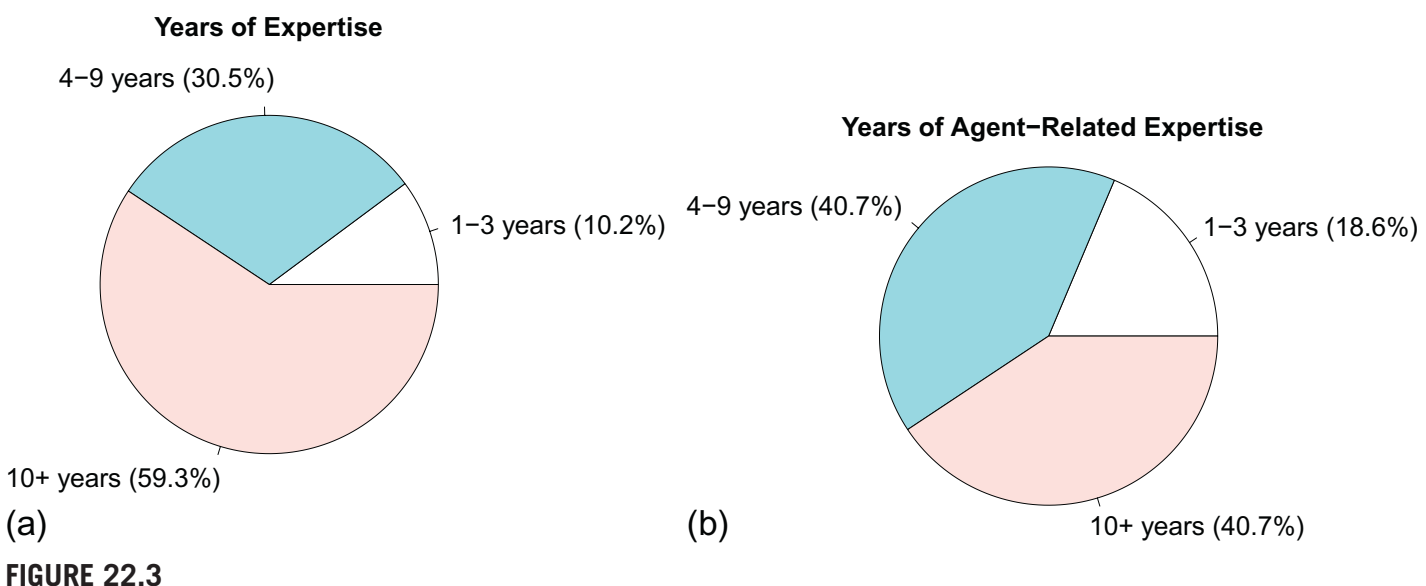

Survey demographics: expertise.

"agree," "neutral," and "disagree" regions, the respective questions of the survey are also depicted on the left side, which may provide further insights.

All results are centered in order to provide an easy understanding and comparative analysis. As we can see, the survey answers are overwhelmingly on the "agree" side, indicating that the majority of the answers were in the region of 4-5 in the Likert scale. Considering the demographics of this survey, this is not surprising. A significant portion of answers also concentrates on the neutral region, with the rest on the "disagree" side.

\subsubsection{INDUSTRIAL AGENT ACCEPTANCE}

Industrial agent acceptance is the main motivating factor for the survey and the analysis that follows. As can be seen from the survey results, illustrated in Figure 22.4, the overwhelming majority of participants consider that, generally, industrial agents pose a promising approach overall. However, there is also wide agreement that their usefulness still needs to be proven in practice. The latter holds especially true for industrial settings because many of the prototypes and demonstrators developed have been done in labs, as reported in the literature, but long-term proof of usefulness is still not adequately demonstrated.

Generally, the survey shows that industrial agents provide added-value when used in productive systems and that the technology and concepts may be adequately mature for many but not all cases. This is in line with technologies that are mature enough, but have not yet managed to establish themselves widely in industry for various reasons.

We can also see that industrial agents seem to complement well other existing approaches, such as web services, cloud computing, and IEC 61499, which implies that they could pose a paradigm that may act as a substitute for other applications. On the contrary though, there is less confidence that the industrial agents concepts can be implemented by non-agent technologies. The latter shows that not all dynamics of industrial agents may be easily substituted by other technologies, which also depicts that industrial agents may offer competitive advantages for specific domains or scenarios that cannot be easily tackled by other non-agent approaches. 


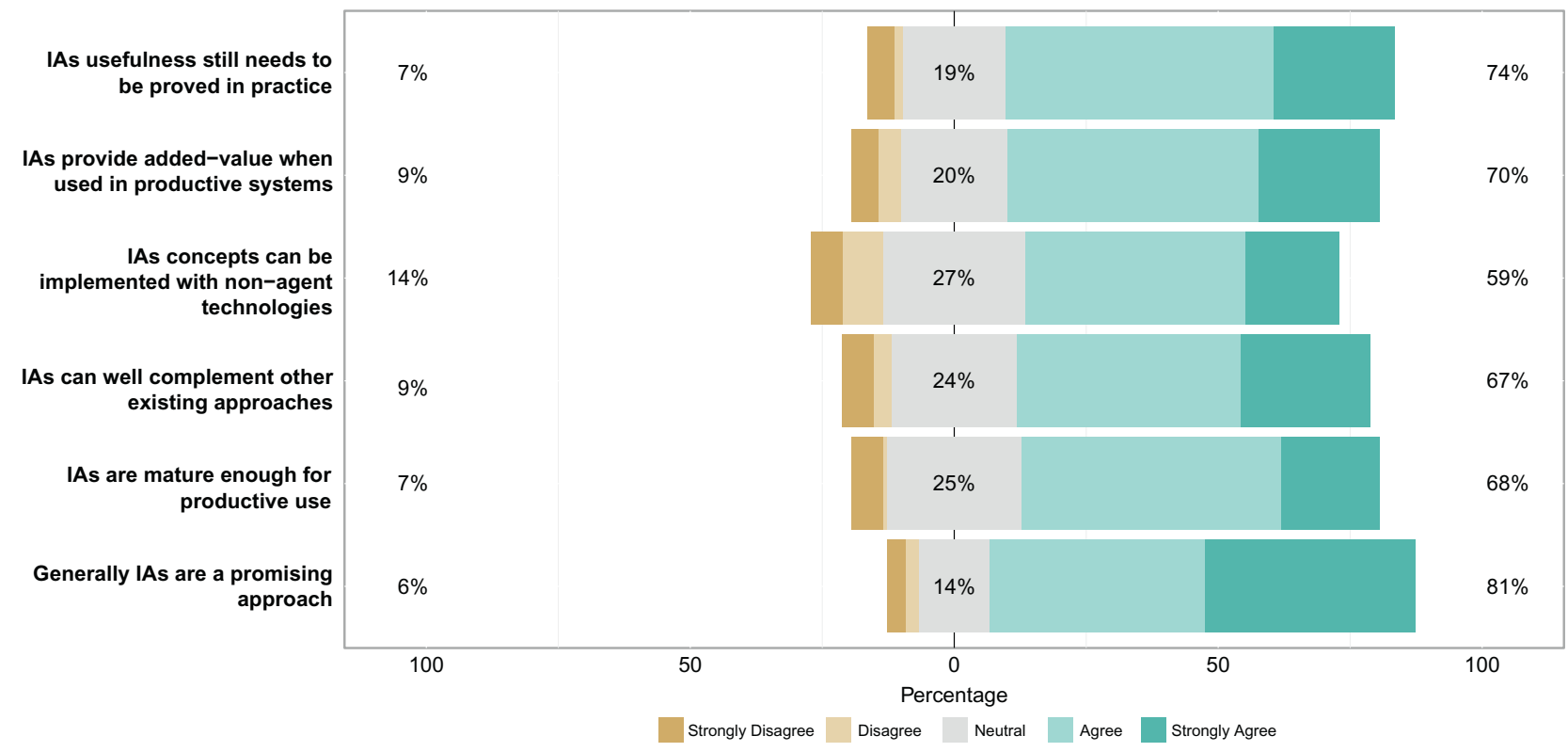

FIGURE 22.4

An industrial agent acceptance overview. 
A more in-depth examination of the dataset reveals some interesting observations. The engineers/ developers seem less confident (69\%) than the managers $(83 \%)$ when considering industrial agents as a promising approach. Sixty-one percent of managers believe that the usefulness still needs to be proven in practice, while a smaller percentage $(11 \%)$ sees no further need to do so. Generally, though, the industry shows strong confidence $(83 \%)$ in industrial agents as a promising approach. Industry also considers industrial agents ready for productive use (79\%), while SMEs are a bit more skeptical (60\%). However, only $55 \%$ of engineers/developers seem to support industrial agents' maturity for production, in contrast to managers $(72 \%)$, researchers $(73 \%)$, and professors $(66 \%)$.

\subsubsection{DESIGN}

A key decision-making factor that relates to the acceptance of industrial agents solutions may be linked to the actual design of such solutions. This can be investigated by analyzing the different characteristics needed design-wise from the industrial agents solution (Figure 22.5).

The overwhelming majority of the survey participants consider that reliability, robustness, and fault-tolerance are important properties for industrial agent solutions and directly link to their acceptance. This comes as no surprise because in industrial environments the real-world solutions are sought to deliver results in a deterministic manner. Formal modeling of industrial agent systems may provide the necessary assurance, especially for more complex systems and systems of systems.

Heterogeneity and interoperability also seem to be key features. Industrial agents that can hide heterogeneity, that are interoperable with existing systems and can "plug-in" to existing infrastructures are sought. As such, results from the industrial agents solution can also easily be integrated and fed into other systems.

Scalability, security, and privacy, as well as distribution and decentralization, are also highly ranked. This implies that there is a need to move beyond monolithic approaches toward solutions for large infrastructures that take advantage of the distributed resources dynamically and scale seamlessly. Industrial agents should be designed with security in mind, which is not trivial (Karnouskos, 2001) considering the distributed and networked nature of the sought industrial agents' design characteristics. It is expected here that industrial agents will be able to tap into the ongoing efforts for security, trust, and privacy approaches developed for distributed and mobile software systems.

There seems to be a lack of best practices for the industrial agents' design, and the survey responses indicate that such practices could enable an overall better design of industrial agents and boost their acceptance. This may call for open community-wide repositories with guidelines/practices for the design and implementation of industrial agents to avoid pitfalls and realize better solutions. However, one has to be careful not to impose limitations to the scope of a practice. Hence, the specific characteristics may need to be decided case by case.

Generally, there is a need for open and standardized interfaces that will enable interaction among disparate systems and act as enablers for cooperation and easy integration. We do not argue here for nonproprietary core parts, but rather for open interfaces when interacting with other entities and as a good practice among the core modules of industrial agents themselves. In fact, agent-based solutions should be provided as black boxes-i.e., hiding the system complexity and showing only their functionalities using standard interfaces. An analogous example of this would be our cars, where complexity is hidden from the driver, who only needs to know the car's key functionalities. 


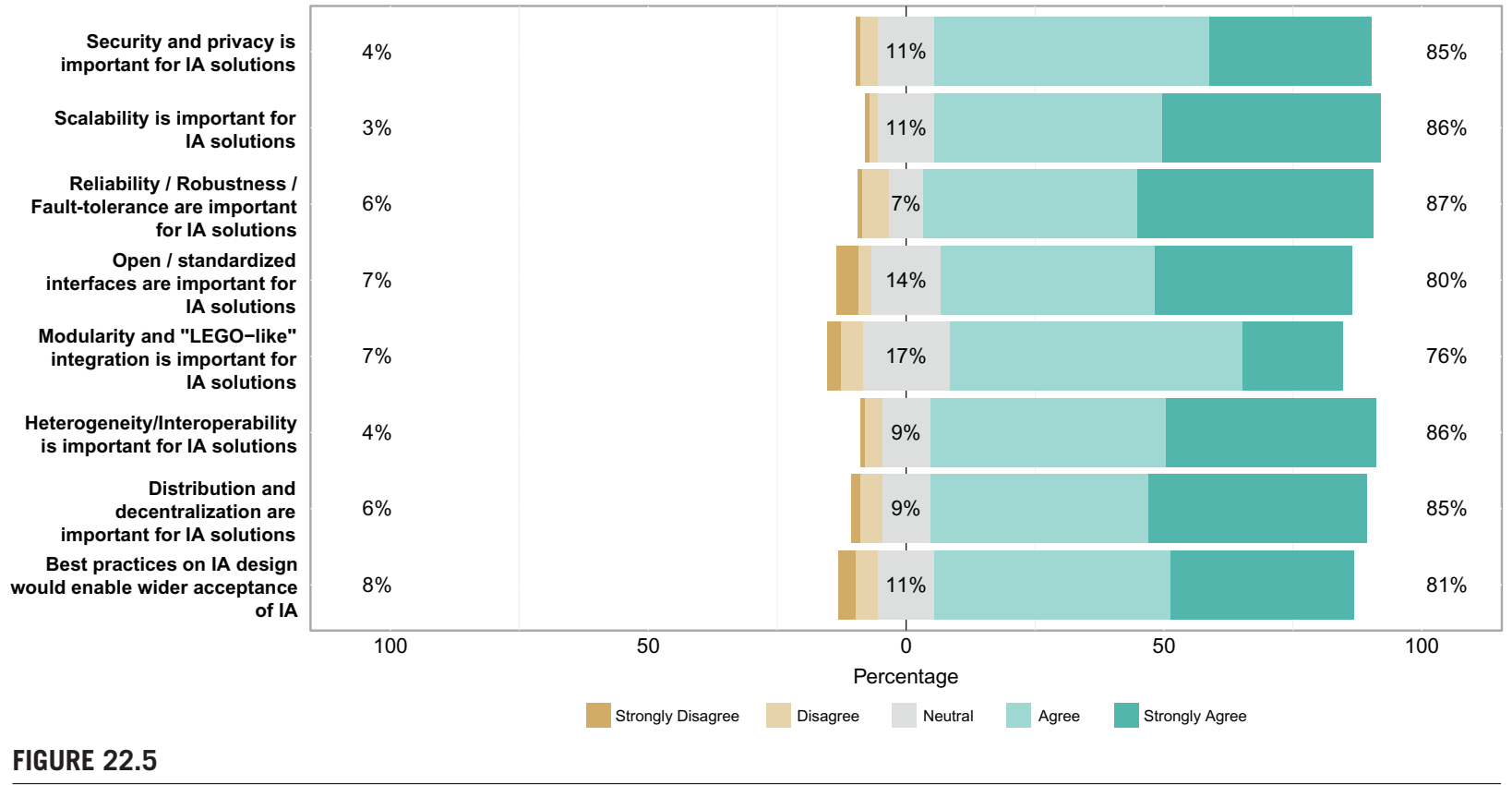

Design factors. 
Today also the development of open interfaces from agents to proprietary devices may further improve industrial agent acceptance. Additionally, standardization, development methodologies, and frameworks are sought that would provide a better basis for understanding, designing, and realizing industrial agent solutions.

Additionally, modularity is sought and "LEGO-like" integration is mostly expected because then solutions can be more easily extended with additional features and also be easily integrable. The modularity is also expected to enable evolution of the different industrial agents' components and may act as the starting point for optimizations-e.g., on computation algorithms and performance.

Finally, we need to point out that although there is a general consensus (as also seen from the survey answers) that all of these characteristics are needed and have an impact on the success of industrial agents, specific industries might weigh them differently based on their requirements and use case. Hence, future research may need to focus on per industry or even specific case scenarios and offer best practices customized to their needs.

A more in-depth examination of the dataset reveals some interesting observations. Security is considered key, with only some lesser percentages-i.e., 7\% in industry and 10\% in SMEs-not considering it important. The latter may be due to specific cases-e.g., internal usage or standalone simulations where security and privacy issues may not be applicable. Mostly researchers (91\%), students (89\%), and engineers/developers (83\%) want best practices for the industrial agents' design, while surprisingly $14 \%$ of industry doesn't seem to value its impact on industrial agent acceptance. Modularity/ LEGO-like integration and scalability are a must for industry (83\%), and are also strongly supported by managers and engineers/developers.

\subsubsection{TECHNOLOGY}

The advanced concepts of industrial agents rely on technology to implement them. As such, it is expected that technology acts as a medium to realize the industrial agents' promised functionality and adherence to requirements for the whole life cycle of the industrial agents solution (Figure 22.6).

As is evident from the survey, modern IDEs are needed. The main role of IDEs is to facilitate the easier development, testing, and deployment of the software solutions, which enables developers to be more productive. It seems that for industrial agents there are only a few such limited environments, and the extension of modern IDEs-e.g., the eclipse or development of new ones to be used in the industrial agents domain - is needed. Nevertheless, IDEs can help with the development and testing phases of the industrial agents' life cycle, and their use may lower barriers and make industrial agents more attractive for industrial applications.

It is also overwhelmingly supported that industrial agents should interact via proven Internet technologies with other systems and among themselves, as this might lead to wider usage of them. This also implies that proprietary technologies should be avoided in favor of interoperability, integration, and interaction. Although it is not always essential to avoid proprietary technologies, following a modular design and using open APIs and technologies for communication and interaction have their benefits. This opens the door to the use of complementary technologies-e.g., by encapsulating agents' functionalities as services being offered and consumed using service-oriented principles through web technologies.

A significant portion of survey participants also bare the view that industrial agents should support multiple technologies (and not be confined with only one) in order to achieve wider use. The latter implies the adoption of the Internet, as well as domain-specific industrial technologies (proprietary or 


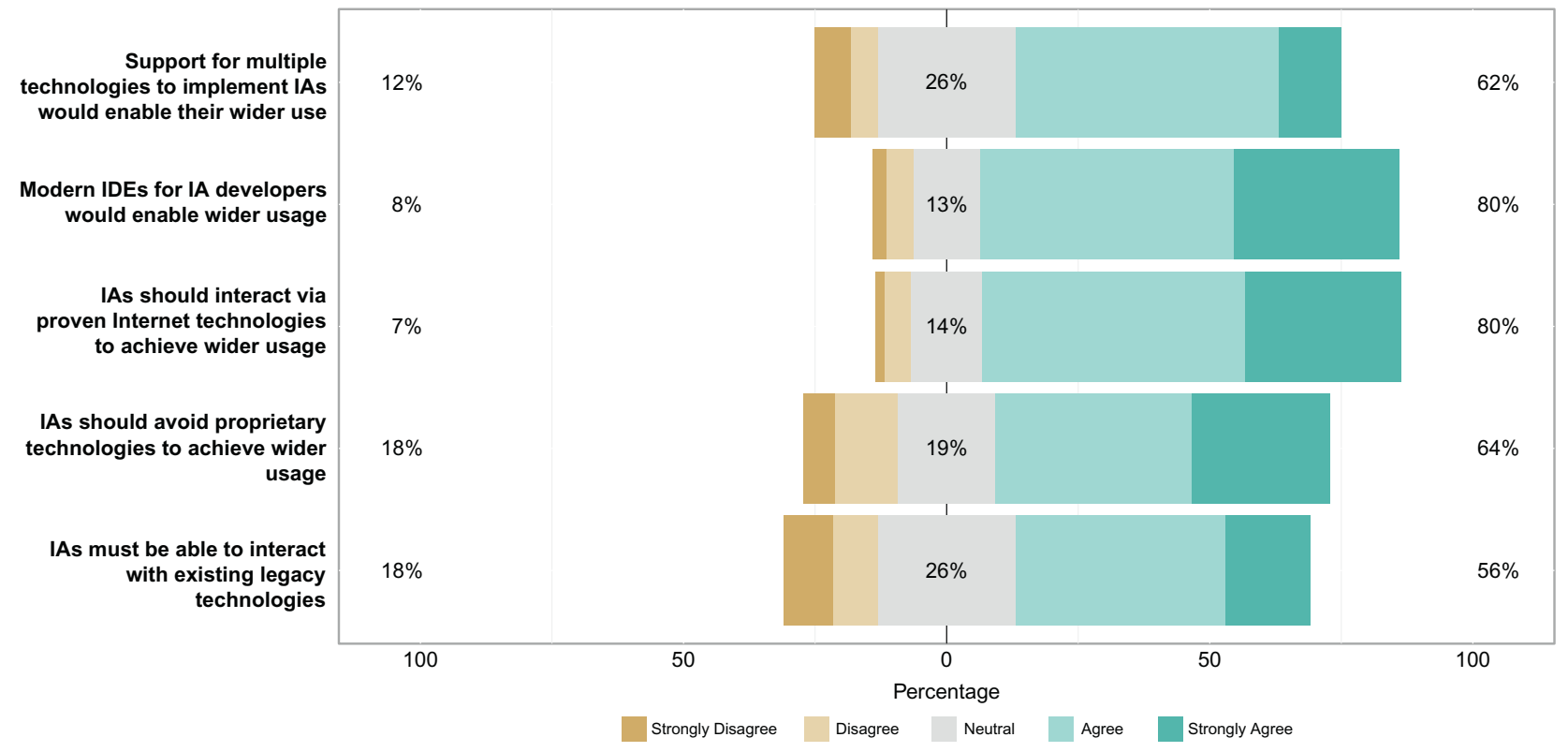

\section{FIGURE 22.6}

Technology factors. 
not). This is also in line with the expectation shared that industrial agents must be able to interact with existing legacy technologies in order to enable seamless integration in existing infrastructures but also be able to assist with the smooth migration toward more advanced infrastructures.

A more in-depth examination of the dataset reveals some interesting observations. There is a pattern showing that with experience, experts give more importance to aspects related to the technology factor. The majority of the industry believes that support for multiple technologies to implement industrial agents would enable their wider acceptance (62\% acceptance, with only $10 \%$ opposition). All experts recognize the importance of the use of proven Internet technologies, demonstrating that we are in the Internet of Things era, but experts with more experience give more importance to it than younger ones. Managers and professors clearly agree with this aspect (none against); however, $14 \%$ of engineers/developers don't agree. Approximately $15 \%$ of experts with 10+ years of experience believe proprietary technologies should be used, which is reinforced by $31 \%$ of engineers/developers.

Experts with more experience recognize the importance of industrial agents being able to interact with existing legacy technologies $(60 \%)$ with a clear evolution of this trend being the increase in years of experience. Managers score this point higher than engineers/developers or researchers, which means that the newest sometimes don't see the need to address this important industrial requirement. This is clearly illustrated in the score of students, only $33 \%$ of whom understand the need to address legacy integration. The use of modern IDEs for industrial agent developers is the aspect that achieved the highest score. In the range of $10+$ years of experience, $90 \%$ recognize this aspect as a major enabler, which clearly shows the lack of available tools.

\subsubsection{HARDWARE}

Industrial agent solutions dealing especially with physical devices and systems may depend on hardware to fulfill their goals. In that sense, integration with hardware and its functionalities may be a factor that impacts the industrial agents' acceptance. The survey participants seem to agree that hardware considerations play such a role (Figure 22.7).

The general view of survey participants is that industrial agent solutions should provide a common API, abstracting from the underlying hardware functionalities. As such, industrial agents are expected to be able to integrate with hardware systems and their functionalities, but then abstract from them and provide their functionalities in a hardware agnostic way. This is in line with the overall views on interoperability and heterogeneity.

The achieved results show the need to interconnect the intelligent software agents with physical hardware devices, recalling the holonic and cyber-physical systems concepts. In such systems, these mechatronic components (called holons or cyber-physical components) comprise a computational part providing monitoring, decision-making, and communication features, and the physical hardware part. Open, transparent, and standard interfaces should be available for this integration purpose, aiming to achieve interoperability in heterogeneous systems.

The survey reveals the need for Industrial agent solutions to run on many hardware platforms, which implies a loose integration with hardware systems. In contrast, less support is witnessed in the survey regarding tight integration, where industrial agent solutions are strongly bundled with specific hardware. Although the latter may still be the case for specialized domains and specific scenarios, the developed solution should not be limited to specific hardware platforms if possible. This clearly points toward the next generation of hardware-enabled but hardware agnostic industrial agents solutions. 


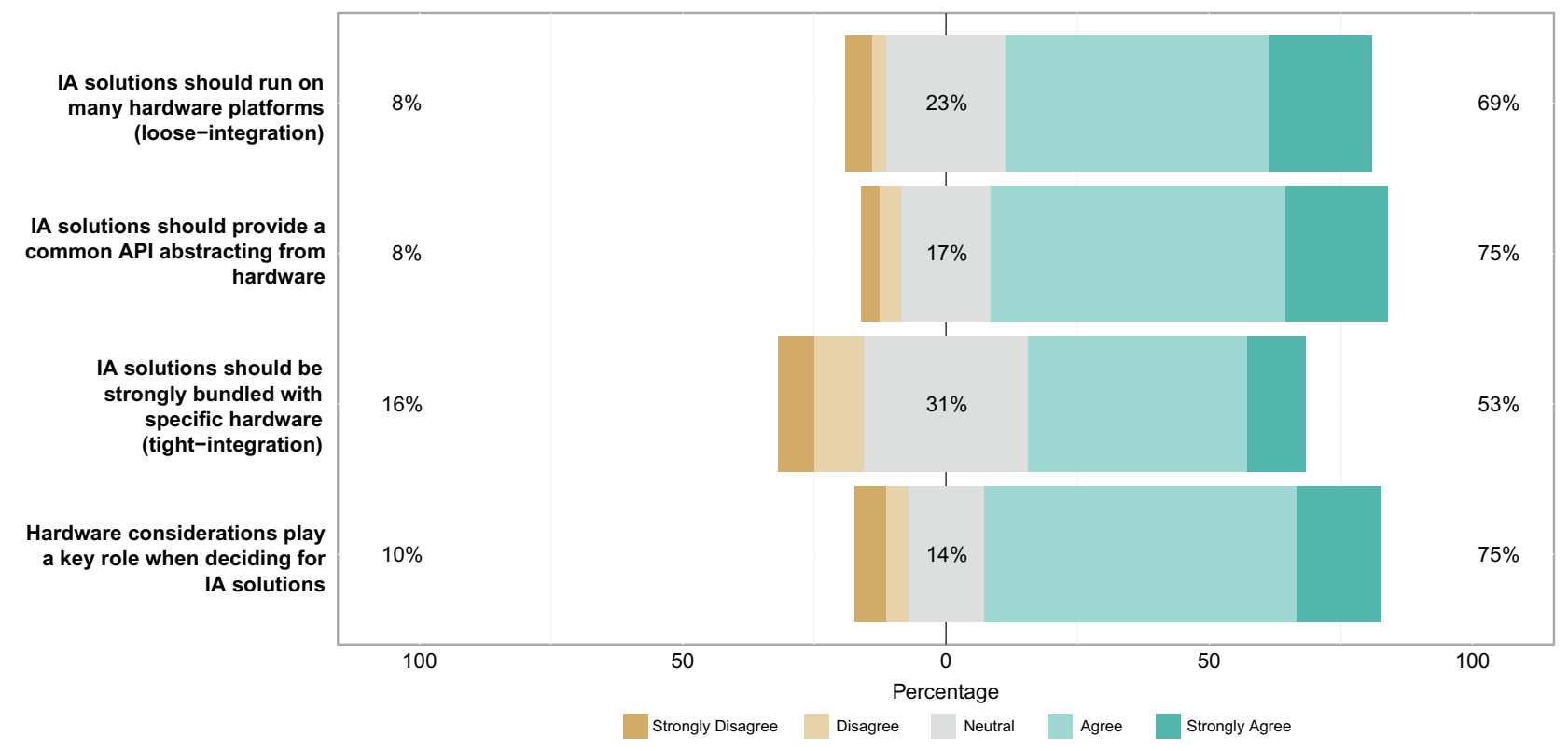

FIGURE 22.7

Hardware factors. 
A more in-depth examination of the dataset reveals some interesting observations. Eighty percent of SMEs, a little less from industry with only $62 \%$, consider hardware an important issue in industrial applications, reinforcing the current trend toward sophisticated cyber-physical systems. A significant number of participants are neutral and do not consider that industrial agent solutions should be strongly bundled with specific hardware (tight integration).

\subsubsection{INTELLIGENCE/ALGORITHMS}

Industrial agents reside on several aspects in order to achieve their goals, and the most important of all is probably the intelligence or algorithms they employ to do so. As such, the sophistication of intelligence and algorithms utilized in industrial agent solutions may be the differentiating factor, leading to their wider acceptance (Figure 22.8).

There is already a wide range of available algorithms. However, the survey participants overwhelmingly consider that new and effective algorithms could further contribute to the success of industrial agents. Effectiveness might be the key here, which also includes other aspects, such as high performance and deterministic behavior. Self-adaptation and autonomous behavior, especially considering the design characteristics of industrial agents, are already a research area that may lead to future industrial agents-based solutions.

It should be possible to easily include new algorithms in a modular manner. We clearly see a need to be able to exchange or tweak key parts of the industrial agent intelligence in order to see how it performs, without having to replicate large portions of its surrounding functionality (e.g., communication). This calls for well-designed and clearly separated logic, as well as interfaces.

The survey also reveals that industrial agent algorithms are not meant to operate in stand-alone mode, but enable collaboration and interaction among agents. This probably stems from the need to model and operate in complex industrial systems, where dynamic situations that arise may need to be tackled collectively. Along the same line of thought, it is believed that algorithms should enable distributed applications and therefore interact with the surrounding environment. Ontologies are still an area of research, and new advances may provide better operation and collaboration among industrial agent solutions and ease their acceptance in industry.

Resource efficiency is an issue, but may not be as critical as other aspects. This does not really come as a surprise in the era of vast computational and communication resources available to industrial agents in order to perform their tasks. Our consideration is that optimizations with respect to resource usage will depend on the specific domain and the overall impact on key system characteristics.

A detailed analysis of the dataset reveals some interesting observations. Surprisingly, $31 \%$ of research center experts disagree that industrial agents should be able to include new algorithms easily in a modular manner, being more conservative than industry and SMEs. This may be due to the fact that in research people develop prototypes usually demonstrating some features and not properly addressing the whole life cycle of it—e.g., maintenance, easy reuse by others, etc. In a similar way, industry and SMEs consider that new and effective algorithms will contribute to the success of industrial agents (79\% and 70\%, respectively). The majority of experts consider that industrial agent algorithms should be resource efficient, but surprisingly $21 \%$ of industry doesn't see this issue as a real need. The latter may be justified with the tremendous resources (communication, computation, memory, storage, etc.) available to modern hardware, as well as the emergence of applications in the cloud. 


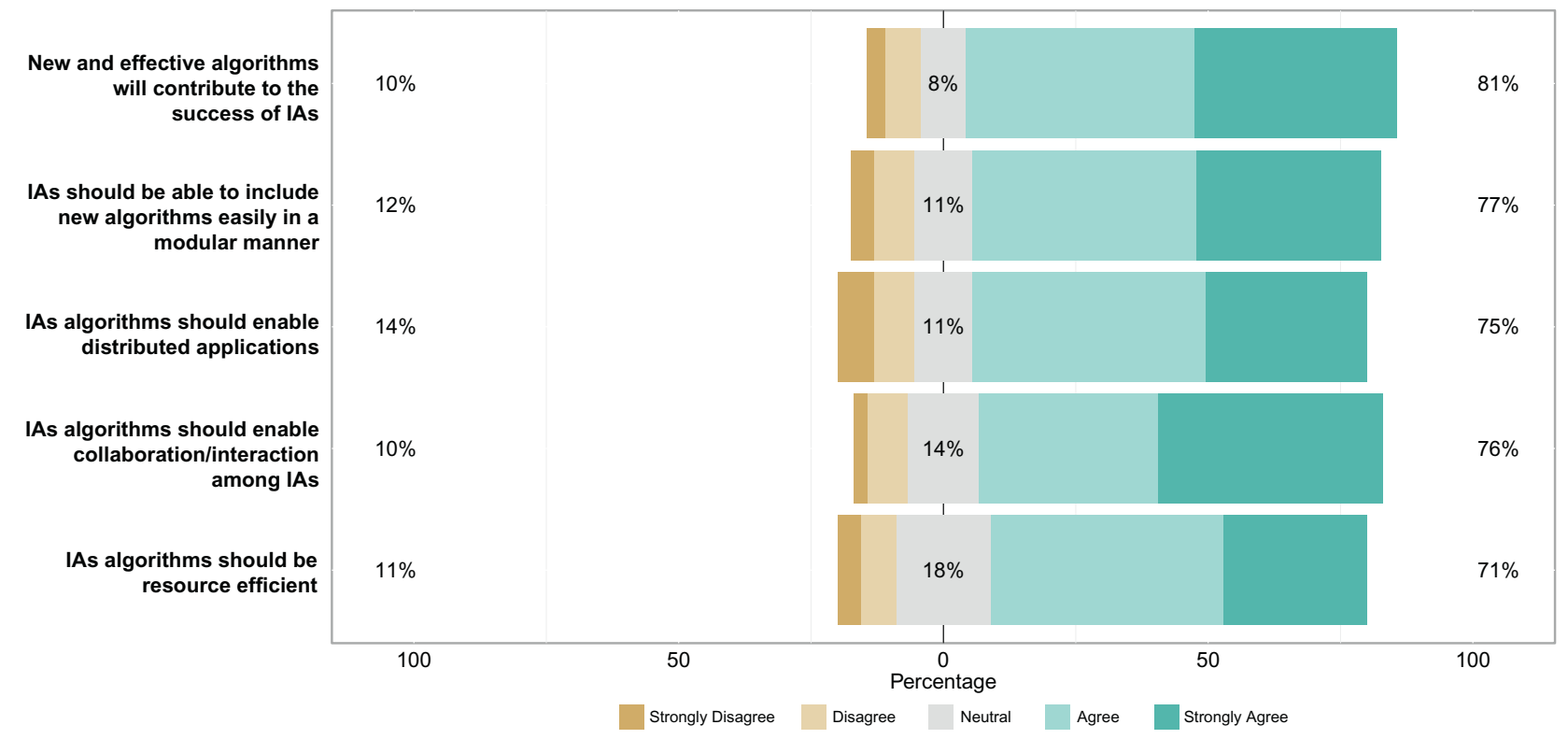

\section{FIGURE 22.8}

Intelligence/algorithms factors. 


\subsubsection{COST TACKLING}

Costs play a key role in any decision-making process, and especially in industry the introduction of new paradigms and technologies must not only be justified context-wise but also cost-wise in the midor long-term. To that end, industrial agent acceptance could be affected by costs associated with their utilization (Figure 22.9).

Some industrial agent solutions require specific hardware and form a strongly coupled solution. It is a common thought that such coupling plays a key role in their acceptance. The required hardware could be broad in nature and could range, for example, from specific monitoring and control equipment, up to simple "server computational needs" required to run a specific simulation. We have to point out though that hardware costs are usually accepted in industry, especially if the solution deals with physical assets. The same though does not hold true for software where high costs are not well seen.

However, apart from hardware costs, other factors are equally important, namely the cost of maintenance and long-term support. These costs may be significantly higher than the initial investment to a solution and are continuous, affecting the mid- and long-term perspectives.

The costs for the development of the software related to industrial agents also seem to play some role. This refers not only to the cost to develop the original industrial agent solution, but also the cost to facilitate the integration with other systems, and the adjustments these will need to undergo in order to fit in the new infrastructure. The latter (i.e., the migration from the existing approaches to the industrial agent solution ones) also plays a role, although the introduction of industrial agents is usually done within the context of a more general migration of a system or process and not only because of the industrial agent solution usage.

Finally, the cost of personnel training may also be relevant, because people will need to understand the new technologies and their capabilities. However, we have to point out that decisions are usually not only made on a technology basis but on the basis of complete solutions, functionalities, and other features that they bring. Additionally, ROI in the short- and mid-term may also play a role in whether an industrial agent solution might be chosen or not (e.g., compared with competitive approaches or other technologies).

A more in-depth examination of the dataset reveals some interesting observations, particularly the results provided by the managers, because they are the stakeholders that will decide the adoption of the technology from the cost perspective. The cost of software development is important but it is not the major aspect to be considered within the cost factor, which are clearly the costs of hardware and maintenance. Managers don't see the cost of hardware affecting the industrial acceptance (only 56\%), but for experts with $10+$ years of experience this aspect gets the higher score (77\%). Managers believe that the cost of initial installation/deployment affects the industrial acceptance (no negative answers); however, they have significant doubts (50\% neutral) and SMEs don't recognize this issue as crucial (80\% and no negative answers).

Managers believe that the cost of maintenance and long-term support affects industrial acceptance (89\% and none against), being also supported by industry. Engineers/developers, researchers, and managers don't give so much importance to the costs related to personnel training for the industrial agent solutions (45-55\%), and SMEs are a little bit more receptive to this issue than industry. Only 50\% of managers consider that the cost of migration to industrial agent solutions affects the industrial acceptance, and only experienced experts are aware of the (usually hidden) migration costs, which means that this can be a bottleneck when the technology is being assessed by experts with lower experience. 


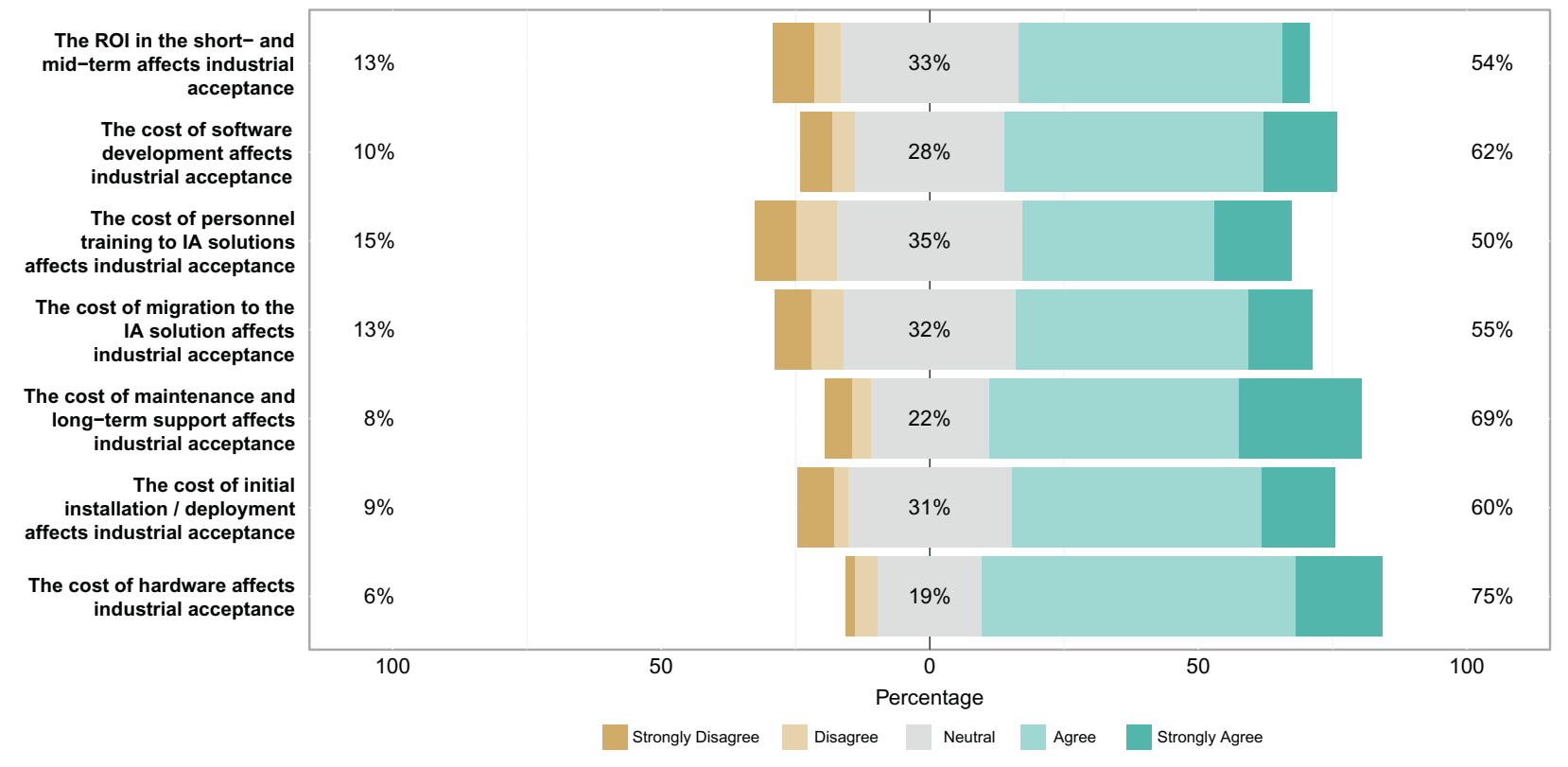

FIGURE 22.9

Cost factors 
We also notice that $22 \%$ of managers don't believe that ROI in the short- and mid-term affects industrial acceptance, and only $61 \%$ believe it does so (this is the lowest score among the organization position groups). This is surprising, unless they think that it only affects the long-term perspective. SMEs are more skeptical than industry and government regarding the ROI in the short- and mid-term. The university and research experts are mainly neutral, reflecting their natural position far from the need to make profit with the developed solutions.

\subsubsection{STANDARDIZATION}

Standardization is a key issue for any kind of technology attempting to establish itself. For industrial agents, this is also the case. There have been several standardization initiatives in the broader agent domain-e.g., FIPA (Foundation for Intelligent Physical Agents), which is a de facto standard for the development of agent-based solutions, as well as satellite technologies defined by entities such as OMG (Object Management Group), IEEE (Institute of Electrical and Electronics Engineers), W3C (World Wide Web Consortium), IEC (International Electrotechnical Commission), OASIS (Organization for the Advancement of Structured Information Standards), and VDE (German Association for Electrical, Electronic \& Information Technologies). Seixas and Leitao (2013) have already highlighted the need for such efforts.

Solutions based on standards seem to be preferred (Figure 22.10). The same holds true for open Internet technology-based solutions. The reasons are, as in all technologies, that they avoid vendor lock-in and provide some guarantees for interoperability and cost control for the future. Especially for industrial applications, the Internet of Things (Höller et al., 2014), as well as cloud-based industrial automation (Leitão, 2009; Colombo et al., 2014) standards seem relevant.

For the industrial agents specifically, there seems to be a need to support industrial automation standards which seem not to be firmly integrated in current industrial agent solutions. As an example, the adoption of OPC-UA (OLE for Process Control - Unified Architecture), which is an industrial automation de facto standard, may contribute to enabling transparent, multi-vendor interoperability, allowing cross-vertical communication among the ISA 95 layers.

In addition, there is strong support for more modern web and Internet standards that need to be integrated into industrial agent solutions. On the other hand, new standards for industrial agents should be developed that integrate these cutting-edge advances.

A detailed analysis of the dataset reveals some interesting observations. The majority of industry considers that solutions based on standards are preferred, constituting an important industrial requirement; however, $30 \%$ of SMEs and $13 \%$ of experts with $10+$ years of experience disagree (surprisingly the youngest experts are more aware of this requirement than older ones). Managers consider that industrial automation standards need to be considered and supported in industrial agent solutions (83\% and no negative answers). The majority of industry, SMEs, and managers recognize the nonexistence of industry standards in practice for industrial agents, but a minority refers the existence of industry standards. Also, $45 \%$ of engineers/developers refer to the missing or inadequate industry standards. The other groups, and particularly the managers, clearly support this idea. Managers consider that open Internet technology-based solutions are preferred (94\% in favor and none against), which is confirmed by SMEs $(80 \%)$. Engineers/developers are skeptical of the adoption of new standards, considering 


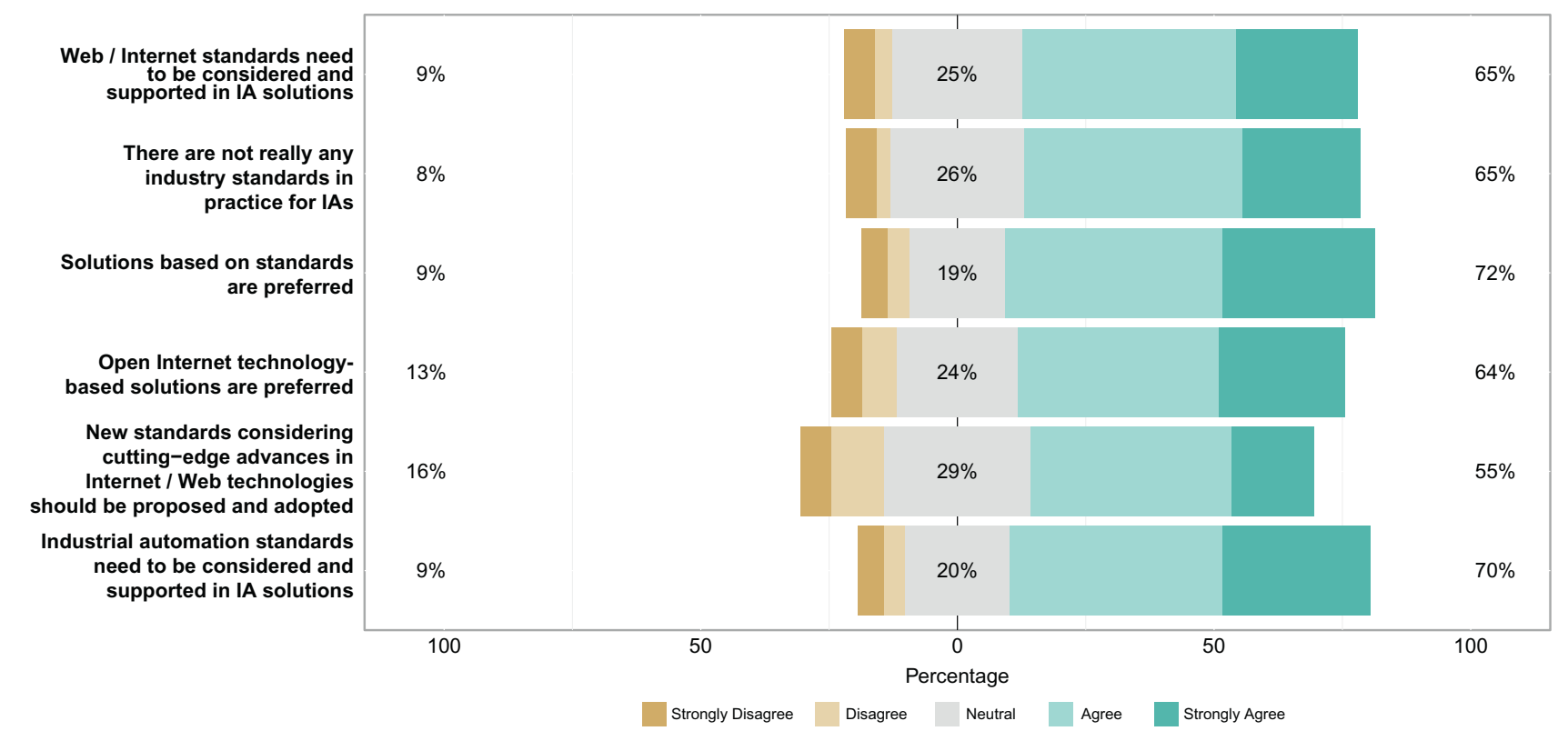

FIGURE 22.10

Standardization factors. 
cutting-edge advances in Internet/Web technologies (38\% against), which is surprising, while managers seem to be more aware of this topic.

\subsubsection{APPLICATION}

The application of industrial agents is heterogeneous and spans many different domains. However, common key utilization cases can be identified, and these mostly deal with simulation or emulation scenarios, the monitoring of systems and processes (all of which are grouped under the broader understanding of assets), as well as various levels of infrastructure control/management (Figure 22.11).

The results show that there is overwhelming support for the utilization of industrial agents for simulation/emulation scenarios-e.g., of assets, systems, and processes. This comes as no surprise because agents in general are competitive simulation tools that can deal with complex behaviors and scenarios. Particularly, agent-based modeling and simulation tools can be suitable used for optimization processes and big data analytics, with agent-based solutions running in cloud or HPC (high-processing computing) platforms. As an example, Repast (Recursive Porous Agent Simulation Toolkit) HPC (Collier and North, 2013) is an agent-based modeling and simulation framework already prepared for massively distributed multi-agent systems solutions over large-scale distributed computing platforms.

Monitoring is also a key case where industrial agents can be utilized, especially when considering them as information collectors from assets, processes, and systems-e.g., by having a direct connection to a production line or by analyzing status messages and logs. In a similar fashion, the majority of survey participants agree that industrial agents can be used to successfully manage assets-a function that well complements the monitoring capabilities.

There are several variations of control that can be applied, which can range from simple reconfigurations (seen as simpler and done via predefined/standardized APIs) to dynamic (even at runtime) reprogramming. However, programming assets (Karnouskos, 2002) requires more in-depth understanding of the underlying assets and goes beyond simple reconfiguration to implement complex and potentially autonomous behaviors.

Although in industry there are several examples of the preceding management/control usage, and controlling real-time assets is seen as a promising use case, there is also considerable skepticism because the requirements to be fulfilled are much more demanding. Real-time control by agents will need additional research to successfully reach the level of confidence that other applications have-e.g., the monitoring ones.

A more in-depth examination of the dataset reveals some interesting observations regarding the application domains that benefit from the usage of industrial agents. The use of industrial agents for monitoring the status of assets and for simulation/emulation of assets/behaviors is seen as the most promising for industry, SMEs, and research centers. Researchers are not as confident in the successful use of industrial agents for reconfiguring assets (only 46\%), and are also skeptical with respect to the successful use of real-time controlling of assets (only 23\%). SMEs see this last application domain as a successful trend, aligned with the opinion of engineers/developers $(69 \%)$. This means that there is a real need for new and better solutions for real-time control, but the use of agent technology at this level, as previously mentioned, remains a problem and requires more research and development to prove its capabilities. 


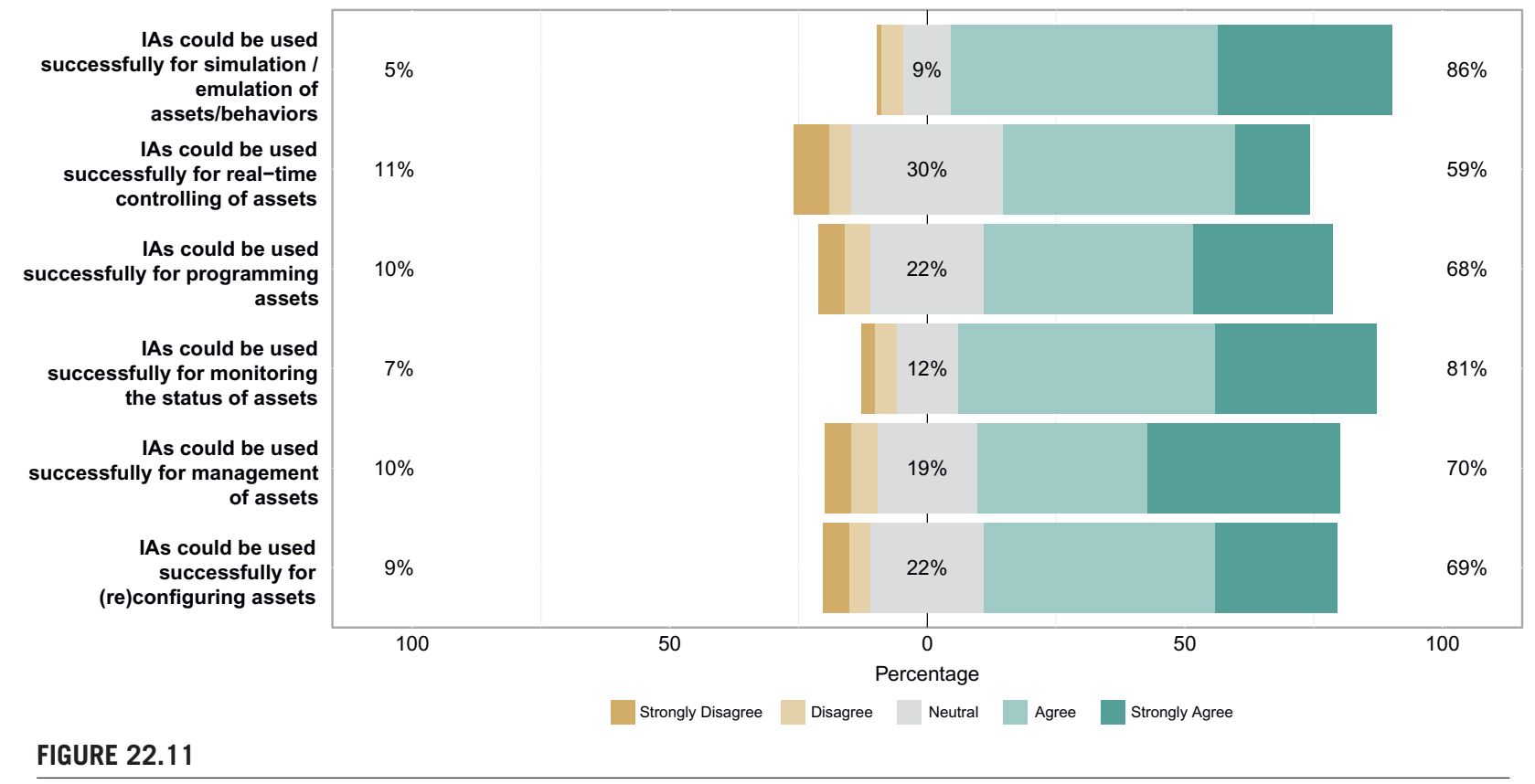

Application factors. 


\subsubsection{CHALLENGES}

As with any emerging approach or technology, its success depends on how well, and sometimes how quickly, several challenges can be tackled effectively. The latter may prevent the approach from reaching critical mass, which would establish it in industry. The same holds true for the industrial agents. Thus, we investigate here such challenges as identified via the survey (Figure 22.12).

As can be witnessed, there is general support from the survey participants that new and better industrial agent technologies need to be developed. This shows that there might potentially be a gap between the industrial agents concepts and the state-of-the-art technologies used to implement them, which is something we look closer at in the technology factor section.

In addition, new and better methodologies and tools should be created. Although we do have mature tools and methodologies, there seems to be a need to advance these further or create new ones. This may imply that the existing ones are too cumbersome or not up to the current standards of modern software engineering solutions for the industry.

Another challenge seems to be the need to prove the business benefit of deploying industrial agents in the real world. This is understandable because most efforts are demonstrable in labs, but longer-term studies of the industrial agent usage seem to be limited. Even then, there is hardly any connection of the technical industrial agents solution and how this impacts the business side and the tangible benefits (or hurdles) it introduced. It is also more explicitly pinpointed that there is a need for economic analysis tools that will help uncover industrial agents' economic impact, especially in complex scenarios, and therefore also build up support cases for industrial agents overall.

The results show some confidence in technology, but there is still room for improvement. This could be strengthened with comparative analysis studies, as well as "success stories" where industrial agents have been used to provide industrial solutions, satisfying the industrial requirements set.

There is a need for benchmarking and comparison criteria for different industrial agent solutions. Today, there is hardly any way to effectively compare various solutions with industrial agent ones, as well as compare industrial agent solutions among themselves. Developing such comparative analysis approaches may enable better understanding of their shortcomings and enhance the acceptance of industrial agent-based solutions.

Although some business models exist, new ones should be developed because existing ones may not be sufficient. This comes as no surprise because the business world is rapidly developing and stateof-the art systems need to be developed-e.g., when dealing with simulations. In addition, business models with respect to industrial agents themselves should also be a priority. The latter could describe the rationale of how the utilization of industrial agents creates, delivers, and captures value. Because industrial agents offer solutions, there is a need to investigate their fit and how they can benefit the core aspects of a business-e.g., business processes, strategies, infrastructure operational processes, customer relations, revenue models, and cost structures.

Opinions on the social-related aspects, including privacy and user-friendliness, diverge, and although they are seen as necessary and should be addressed, many consider them to be something further down the road rather than an immediate concern. As an example, visualizing industrial agents' behaviors so that plant operators can understand their logic and so actions may increase user-friendliness and have a positive impact on the industrial agents' acceptance.

As we have seen, there are several high-level challenges that need to be dealt with. The survey reveals that if this is done adequately, there might be a positive impact on the industrial agents' acceptance 


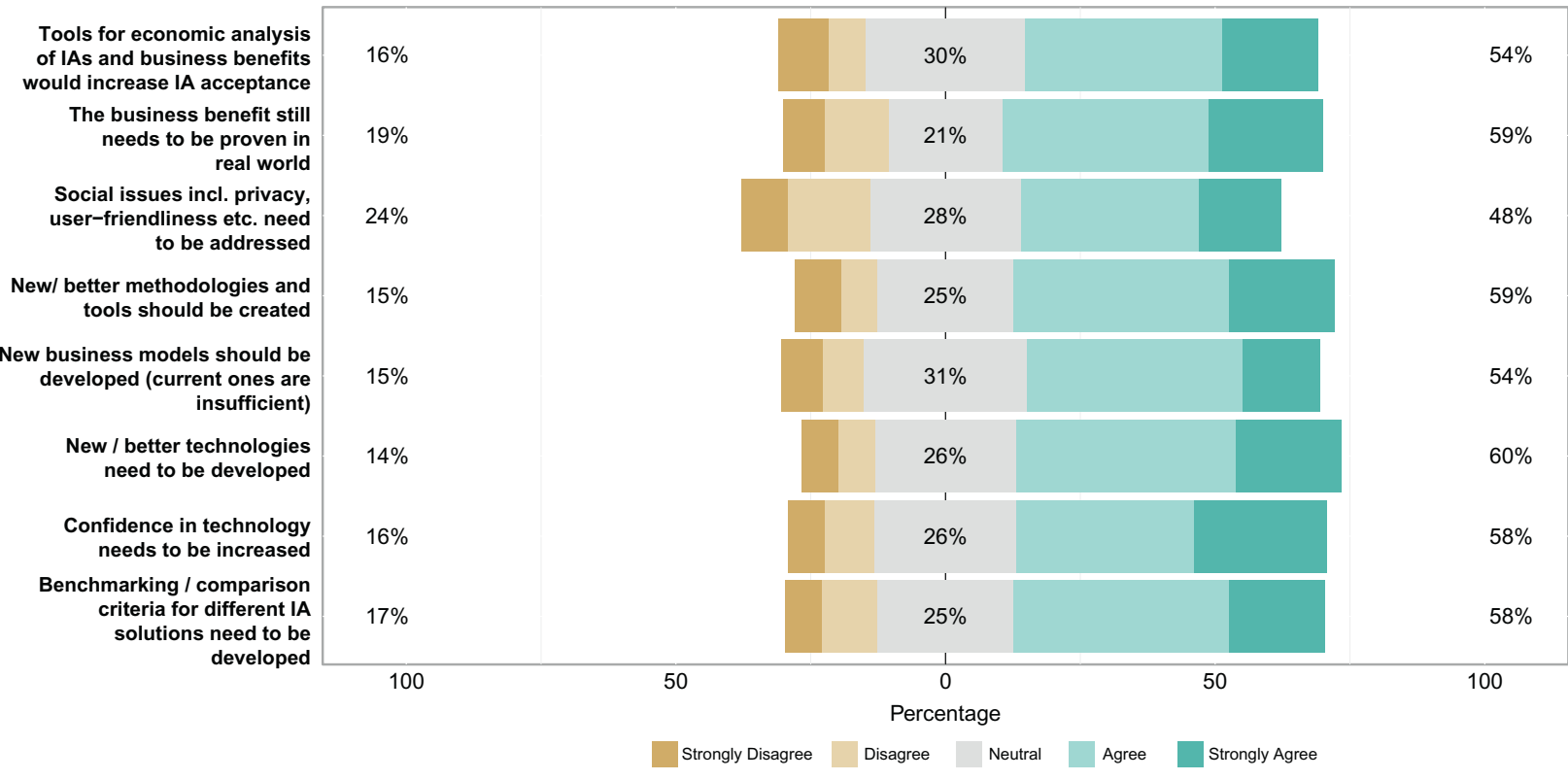

FIGURE 22.12

Challenges factors. 
in industry. We also witness many requirements that go beyond technical needs and pose a clear call for cross-disciplinary research between industrial agents, economics, and business.

A detailed analysis of the dataset reveals some interesting observations. Although $61 \%$ of managers think that new business models are needed (none against), engineers/developers believe that current ones are sufficient. Managers point out that confidence in technology needs to be increased (72\%), but $24 \%$ of industry overall seems to already be convinced of the agent technology itself, which is a positive signal, but highlights the need for a long path to convince industry stakeholders of the tangible benefits of industrial agent technology. In fact, managers are those that are more convinced that business benefits still need to be proven in the real world; however, $17 \%$ of managers and $28 \%$ of the industry are convinced that benefits have already been shown in the real world, showing that they are open to adopting agent technology. The achieved results show that the need for new/better technologies is not a main challenge, which means that it is more important to explore the existing technologies instead of developing totally new ones. Engineers/developers highlight the importance of using benchmarking/ comparison criteria, but managers are not aware of this aspect (only $44 \%$ agree, with $44 \%$ neutral).

\subsection{CONCLUSIONS}

The survey described in this chapter aimed to identify and investigate in detail the factors that impact the industrial adoption of agent technology. We have identified and focused on several factors-i.e., design, technology, intelligence/algorithms, hardware, cost, standardization, application and challenges. All of these positively contribute to the adoption of industrial agents, and as we have already discussed, it is clear where the focus should be for future efforts.

Based on the survey results, we can derive an initial set of actions that should be followed to increase the adoption of agent technologies by industry and enjoy the benefits it offers. Such actions include:

- Providing industrial agent-based solutions as black boxes-i.e., hiding the system complexity and showing only their functionalities.

- Providing new methodologies and development technologies that better fit industrial requirements.

- Providing transparent process reengineering, showing that the implementation can be performed smoothly and be compliant with the standards in the field.

- Providing standard APIs to simplify the integration of such systems with legacy systems and physical hardware devices.

- Providing bridges to consider the integration with other complementary technologies, such as web services to address the interoperability issues, with the aim of achieving vertical and horizontal integration.

- Providing demonstrators/solutions running in industry that show the maturity, flexibility, and robustness of the technology.

- Providing trusted ROI analysis, considering the development, installation, and maintenance costs.

The analysis of the survey results allows us also to derive and synthesize the main conclusions using a SWOT model, which aims to identify the key internal and external factors that are important to achieve the target objective. The internal attributes are related to the strengths and weaknesses, and the external factors are related to the opportunities and threats involving business models and that include 


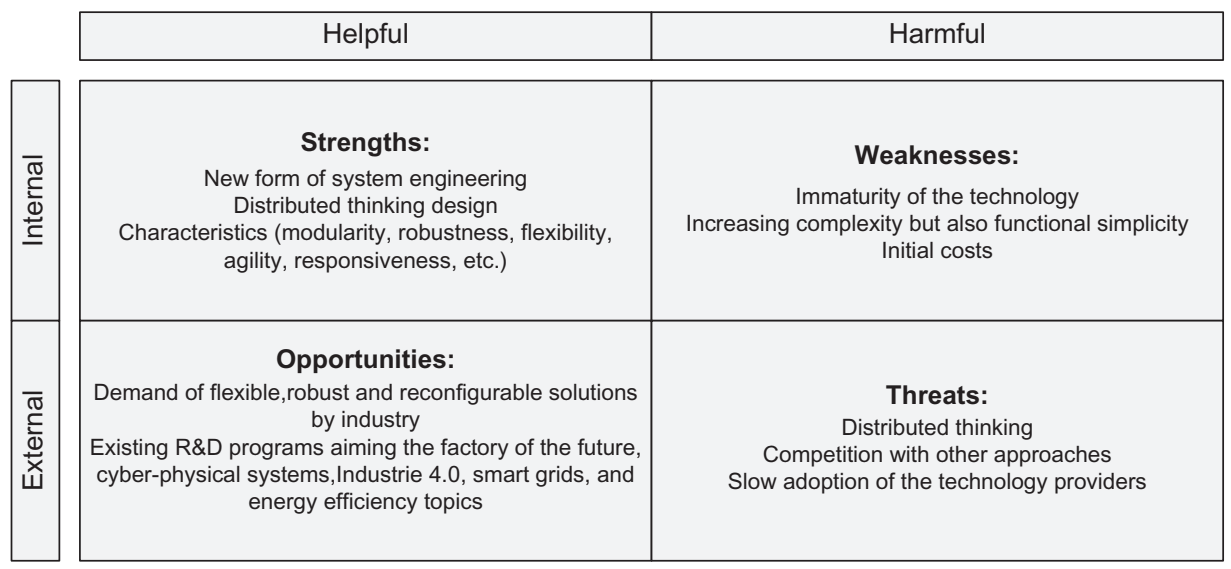

FIGURE 22.13

The SWOT matrix for the industrial adoption of the agent technology.

technological, legislative, sociocultural, and marketplace or competitive position changes. Figure 22.13 summarizes the SWOT analysis in the form of a matrix.

The strengths are the inherent characteristics of the business or team that give it an advantage over others in the industry. In this work, they are related to the positive characteristics of the agent technology, which are mainly the new form of system engineering, the distributed thinking design, and some important characteristics provided by the paradigm, such as modularity, robustness, flexibility, agility, and responsiveness.

The weaknesses are the negative aspects or characteristics that place a disadvantage relative to others, which here are mainly the immaturity of the agent technology, the complexity of agent-based solutions, and the initial costs (as well as the maintenance costs).

Opportunities are external chances to improve the profits in the environment, being in this case related to those that can support a wide applicability of the agent technology in industrial applications. The demand for flexible, robust, and reconfigurable solutions by industry, the existing R\&D programs aimed at the factory of the future, cyber-physical systems, Industrie 4.0, smart grids, and energy efficiency topics, can be pointed to as opportunities for the adoption of agent technology in industrial environments.

Threats are external elements in the environment that could cause trouble for the business. In the case of agent technology, these are mainly distributed thinking, competition with other approaches, and the slow adoption of the technology providers.

Although the agent technology has been with us some decades now, its acceptance in industry was limited. As such, we investigated the main factors that impact it and found that a decision on agent utilization in productive systems is a complex undertaking. There are still many issues to be resolved in the domain in order to lead to a wider acceptance of industrial agents. However, as we have already discussed, the latest developments in the amalgamation of enterprise and industrial networks, the prevalence of Internet technologies in industrial settings, the emergence of the Internet of Things in conjunction with service-oriented architectures (SOAs) and cloud systems, distributed intelligence, as well as huge leaps in increasing computation, communication, and networking in the infrastructure, even 
down to simple sensors and actuators, may provide a second chance for industrial agents. Industrial agents benefit from these advancements and their concepts and technologies can very well contribute toward viable solutions in industry-hence, we may see in the next few years a Renaissance of them in Industry, assuming of course that some key issues are tackled efficiently.

\section{ACKNOWLEDGMENTS}

The authors would like to thank all participants who contributed to the survey questionnaire, particularly the expert members of the IEEE IES Technical Committee on Industrial Agents (tcia.ieee-ies.org).

\section{REFERENCES}

Babiceanu, R., Chen, F., 2006. Development and applications of holonic manufacturing systems: a survey. J. Intell. Manuf. 17, 111-131.

Collier, N., North, M., 2013. Parallel agent-based simulation with repast for high performance computing. Simulation 89, 1215-1235.

Colombo, A.W., Bangemann, T., Karnouskos, S., Delsing, J., Stluka, P., Harrison, R., Jammes, F., Lastra, J.M. (Eds.), 2014. Industrial Cloud-based Cyber-Physical Systems: The IMC-AESOP Approac. Springer, UK, ISBN: 978-3-319-05623-4.

Gartner, 2014. Gartner's 2014 Hype Cycle for Emerging Technologies Maps the Journey to Digital Business. http://www.gartner.com/newsroom/id/2819918 (accessed 08.09.14).

Göhner, P. (Ed.), 2013. Agentensysteme in der Automatisierungstechnik. Springer, UK, ISBN: 3642317677.

Höller, J., Tsiatsis, V., Mulligan, C., Karnouskos, S., Avesand, S., Boyle, D., 2014. From Machine-to-Machine to the Internet of Things: Introduction to a New Age of Intelligence. Elsevier, Amsterdam.

Karnouskos, S., 2001. Security implications of implementing active network infrastructures using agent technology. Comput. Netw. 36 (1), 87-100 Elsevier.

Karnouskos, S., 2002. Realization of a secure active and programmable network infrastructure via mobile agent technology. Comput. Commun. 25 (16), 1465-1476 Elsevier.

KREAagentuse, 2014. Design, Implementation and Evaluation of a Tool-supported Method for the Development of Agent Systems in Automation Technology, Taking into Account the Usability”. DFG project VO 937/8-1, http://www.ais.mw.tum.de/en/research/research-projects/kreaagentuse/ (accessed 22.08.14).

Leitão, P., 2009. Agent-based distributed manufacturing control: a state-of-the-art survey. Eng. Appl. Artif. Intell. 22 (7), 979-991.

Leitão, P., Marik, V., Vrba, P., 2013. Past, present, and future of industrial agent applications. IEEE Trans. Ind. Inf. 9 (4), 2360-2372.

Luck, M., McBurney, P., Shehory, O., Willmott, S. (Eds.), 2005. Agent Technology: Computing as Interaction A Roadmap for Agent-based Computing. AgentLink III, September.

Marik, V., Lazansky, J., 2007. Industrial applications of agents technologies. Control. Eng. Pract. 15, 1364-1380.

Marik, V., McFarlane, D., 2005. Industrial adoption of agent-based technologies. IEEE Intell. Syst. 20 (1), 27-35.

McFarlane, D., Bussmann, S., 2000. Developments in holonic production planning and control. Int. J. Prod. Plann. Control 11 (6), 522-536.

Monostori, L., Váncza, J., Kumara, S., 2006. Agent-based systems for manufacturing. Ann. CIRP 55/2, 697-720.

Müller, J., Fischer, K., 2014. Application impact of multi-agent systems and technologies: a survey. In: Shehory, O., Sturm, A. (Eds.), Agent-Oriented Software Engineering. Springer, Berlin Heidelberg, pp. 27-53. 
Pechoucek, M., Marik, V., 2008. Industrial deployment of multi-agent technologies: review and selected case studies. Auton. Agent. Multi-Agent Syst. 17 (13), 397-431.

Pereira, A., Rodrigues, N., Leitao, P., 2012. Deployment of multi-agent systems for industrial applications. In: Proceedings of the 17th IEEE Conference on Emerging Technologies and Factory Automation (ETFA). pp. 17-21, September.

Schild, K., Bussmann, S., 2007. Self-organization in manufacturing operations. Commun. ACM 50 (12), 74-79.

Seixas, I., Leitao, P., 2013. Standards compliance in industrial agents applications. In: Proceedings of the 39th Annual Conference of the IEEE Industrial Electronics Society (IECON'13), 10-13 Nov. 2013, pp. 7446-7451.

Shen, W., Wang, L., Hao, Q., 2006. Agent-based distributed manufacturing process planning and scheduling: a state-of-the-Art survey. IEEE Trans. Syst. Man Cybern. Part C Appl. Rev. 36 (4), 563-577.

VDI/VDE, 2010. Verein Deutscher Ingenieure (VDI) Verband Der Elektrotechnik Elektronikinformationstechnik (VDE) standard VDI/VDE 2653 Part 1, "Multi-agent Systems in Industrial Automation". http://www.vdi.eu/ nc/guidelines/vdivde_2653_blatt_1-agentensysteme_in_der_automatisierungstechnik_grundlagen/. 